\title{
POROCARCINOMA IN MALE BREAST
}

Lilian de Sá Paz Ramos ${ }^{1,2}$, Juliana Almeida Frank³, Suzana Imbassahy de Sá Bittencourt Câmara e Silva3, Diogo Silva Almeida ${ }^{3}$

${ }^{1}$ Programa de Pós-Graduação em Ciências da Saúde: Ginecologia e Obstetrícia, Universidade Federal do Rio Grande do Sul Porto Alegre (RS), Brazil.

${ }^{2}$ Hospital Aristides Maltez - Salvador (BA), Brazil

${ }^{3}$ Liga Acadêmica de Cirurgia da Bahia, Universidade Federal da Bahia - Salvador (BA), Brazil.

Introduction: Porocarcinoma (PC) is a malignant neoplasm of the eccrine sweat glands, corresponding to $0.005 \%$ to $0.001 \%$ of skin tumors. There are reports of only two cases with primary localization on the breast from a total of 206 cases of pororcarcinoma according to a systematic review conducted by Nazeemi et al. (2018), from 1963 to 2017. The most common anatomical locations are the lower limbs, the head and the neck. This pathology affects elderly individuals and has a similar incidence among genders. This malignant neoplasm usually presents as a single nodule or a plaque with a verrucous or ulcerated surface, sometimes there is a long history of evolution. The most common site of metastases is regional lymph nodes. The pathogenesis of PC is uncertain. This neoplasm originates from the terminal cells of the intradermal segment of the sweat gland called acrosyringeum. In the histological study, the porocacinoma cells may be restricted in the epidermis or infiltrate the entire dermis, the epithelial proliferation of intradermal tumor cells in nests causes acanthosis of the epidermis and hyperkeratosis, cords and polygonal tumor cells proliferate in the dermis with figures of mitosis and areas of necrosis, often ductal differentiation with intracytoplasmic lumina. Immunohistochemical shows positivity for carcinoembryonic antigen (CEA), cytokeratin (CK), pancytokeratin and CK5/6, epithelial membrane antigen (EMA), p53, p63 and CD117. The main treatment is local resection with margins. Sentinel lymph node biopsy can be considered for patients without palpable ganglion, and the performing axillary lymphadenectomy in the context of regional lymphadenopathy. Adjuvant chemotherapy and radiotherapy can be performed in cases of metastatic PC and local recurrence.

Case report: An 82-year-old man presenting with a skin lesion on his right breast with progressive growth, associated with local discomfort and bleeding over two years. He presented a large vegetative, hyperchromic, ulcerated bleeding and painless tumor in the right breast, with an extension beyond the inframammary fold, measuring about $8 \mathrm{~cm}$ in diameter and ipsilateral axillary lymphadenopathy. No evidence of metastasis on chest and in abdominal tomography. The incisional biopsy showed porocarcinoma, the surgical treatment performfed was mastectomy and axillary lymphadenectomy. The histological study revealed an undifferentiated keratinizing carcinoma of the skin, infiltrating the mammary parenchyma, associated with angiolymphatic infiltration and the presence of necrosis and ulceration with free margins, in addition to two metastatic axillary lymph nodes. An immunohistochemical analysis revealed positive cells for EMA, p63, CKAE1AE3 and a K167 proliferation index of 60\% confirming the diagnosis of pororcarcinoma. Local treatment was supplemented with adjuvant radiotherapy. 